$\stackrel{W}{=}$

Global burnals Inc.

है 20 and

\title{
Dietetic Management of Lipid Profile in an Adult Population from the Italian Region of Abruzzo: Role of Mediterranean Diet
}

By Maria Alessandra Gammone, Stefania Martelli, Antonella Danese \& Nicolantonio D’Orazio

Abstract-Background: There has long been a lot of debate about the role of nutrition in the pathogenesis of cardio- vascular diseases. Monounsaturated and polyunsaturated fatty acids, especially n-3 PUFAs are the types of fat that favor metabolic markers and represent central components of the Mediterranean diet, which is considered an ideal dietary pattern with great cardioprotective effect.

Aim: This study aims to assess the influence of Mediterranean diet on lipid metabolism, compared to notMediterranean hypocaloric dietary patterns.

Materials and Methods: This prospective clinical trial evaluated total cholesterol, LDL, HDL, and triglycerides and their modifications in a group of adults in relation to the two different kinds of diet: on the one hand the typical western dietetic pattern, characterized by higher intakes of red meat, dairy products and refined grains, low consumption of fruits and vegetables (L-diet), and the Mediterranean diet (M-diet).

Keywords: mediterranean diet, weight loss, lipid profile, nutrition, cardiovascular risk.

GJMR-K Classification: NLMC Code: WK 819

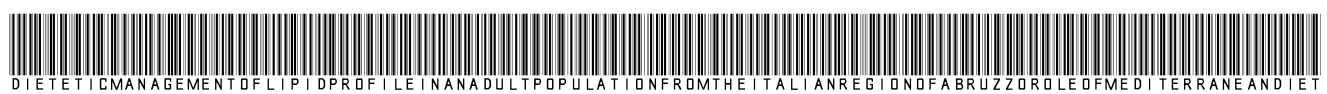

Strictly as per the compliance and regulations of:

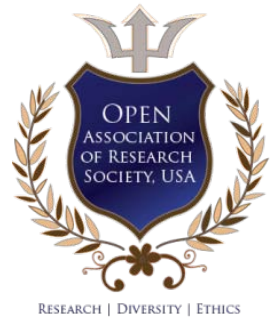

(C) 2019. Maria Alessandra Gammone, Stefania Martelli, Antonella Danese \& Nicolantonio D'Orazio. This is a research/review paper, distributed under the terms of the Creative Commons Attribution-Noncommercial 3.0 Unported License http://creative commons.org/licenses/by-nc/3.0/), permitting all non commercial use, distribution, and reproduction inany medium, provided the original work is properly cited. 


\title{
Dietetic Management of Lipid Profile in an Adult Population from the Italian Region of Abruzzo: Role of Mediterranean Diet
}

\author{
Maria Alessandra Gammone ${ }^{\alpha}$, Stefania Martelli ${ }^{\circ}$, Antonella Danese ${ }^{\rho}$ \& Nicolantonio D'Orazio ${ }^{\omega}$
}

\begin{abstract}
Background: There has long been a lot of debate about the role of nutrition in the pathogenesis of cardiovascular diseases. Monounsaturated and polyunsaturated fatty acids, especially n-3 PUFAs are the types of fat that favor metabolic markers and represent central components of the Mediterranean diet, which is considered an ideal dietary pattern with great cardioprotective effect.

Aim: This study aims to assess the influence of Mediterranean diet on lipid metabolism, compared to not-Mediterranean hypocaloric dietary patterns.
\end{abstract}

Materials and Methods: This prospective clinical trial evaluated total cholesterol, LDL, HDL, and triglycerides and their modifications in a group of adults in relation to the two different kinds of diet: on the one hand the typical western dietetic pattern, characterized by higher intakes of red meat, dairy products and refined grains, low consumption of fruits and vegetables (L-diet), and the Mediterranean diet (M-diet).

Serum levels of total cholesterol, LDL, HDL, triglycerides were assessed in 40 participants (two groups of twenty): ten men and ten women aged between 35 and 45 years. The anthropometric evaluation included the following parameters: weight, height, waist circumference, hip circumference, waist-hip ratio (WHR) and body mass index (BMI). There were anthropometric and hematochemical assessments after three and six months.

Each recruited patient received personalized diets after estimating the correct daily caloric needs through HarrisBenedict Equation. Overweight patients underwent hypocaloric diets with a slight daily physical activity (a thirtyminute walk).

Results: L-diet group showed a slight weight loss for subjects with $\mathrm{BMI}>24.9$, with a mild improvement in total cholesterol levels but a worsening in HDL levels. In the M-diet group, we observed not only a more consistent weight loss in subjects with $\mathrm{BMI}>24.9$ but also a significant improvement in HDL.

Conclusion: The Mediterranean diet was associated to the normalization of the plasma levels of total cholesterol, LDL, $\mathrm{HDL}$, and triglycerides, as well as to an important reduction of body weight and visceral fat, and consequently cardiovascular risk factors.

Keywords: mediterranean diet, weight loss, lipid profile, nutrition, cardiovascular risk.

Author a $\sigma \rho \omega:$ Human and Clinical Nutrition Unit, Department of Medical, Oral and Biotechnological Sciences, University G. D'Annunzio, Chieti (CH), Italy.e-mail: m.alessandra.gammone@gmail.com

\section{INTRODUZIONE}

stituzioni pubbliche e organismi scientifici hanno dato vita, nei vari paesi del mondo, a linee guida o direttive alimentari per indirizzare il consumatore verso una dieta sana e bilanciata. II modello alimentare più diffuso a livello internazionale è la dieta mediterranea, frutto di numerosi studi epidemiologici e sperimentali che testimoniano l'azione protettiva da essa svolta contro patologie croniche degenerative. I paesi del Mediterraneo infatti hanno tassi di morbilità più bassi per malattie croniche soprattutto cardiovascolari e dunque una speranza di vita più alta [1].

Negli ultimi decenni sono stati pubblicati innumerevoli studi sui benefici di essa e di alcune delle sue componenti sulla salute; dimostrando effetti benefici sulle malattie cardiovascolari, sul diabete, sulle demenze, su alcune forme di cancro ed anche sulla depressione [2-5]. È risultata efficace soprattutto per la prevenzione ed il trattamento del sovrappeso e dell'obesità, il cui aumento nel Mediterraneo è conseguenza di una generale riduzione dell'attività fisica sia nell'età evolutiva che in età adulta [6]. La Conferenza Internazionale sulle diete del Mediterraneo tenutasi a Londra nel gennaio del 2000, ha definito come "dieta mediterranea" I'insieme dei cibi tipici utilizzati agli inizi degli anni ' 60 in alcune regioni del Mediterraneo come Creta, alcune regioni della Grecia e il Sud dell'Italia [7], con evidenti effetti protettivi nei confronti di patologie cardiovascolari e tumori. Essa è, pertanto, l'unico modello alimentare al mondo ad essere riconosciuto "Patrimonio dell'Umanità" (UNESCO, Nairobi in Kenya, 16 Novembre 2010) e ad essere definito come un sistema radicato nel pieno rispetto del territorio e della sua biodiversità, che assicura la conservazione e lo sviluppo delle attività tradizionali, artigianali, di pesca e raccolta nelle comunità del Mediterraneo come per esempio quelle di Soria in Spagna, Koroni in Grecia, Cilento in Italia e Chefchaouen in Marocco. Lo scienziato Ancient Keys studiò dal 1950 al 1970 intere popolazioni che contrapponevano nella dieta diversi stili di vita stabilendo quali fenomeni culturali, diete e attività, erano le cause principali dei tassi differenti della malattia di cuore fra la popolazione. Agli inizi degli anni '50 egli viaggiò in diversi Paesi e si accorse che, dove i livelli di colesterolemia erano bassi, i medici ospedalieri locali 
riferivano rarità dell'infarto miocardico. Egli notò inoltre che le malattie cardiovascolari erano poco frequenti a Creta e in alcune aree d'Italia, come nel Cilento, nonostante l'alto consumo di grassi, in particolar modo dell'olio extravergine d' oliva. Sua moglie si dilettò a misurare periodicamente le sue concentrazioni ematiche di colesterolo trovandole basse con rare eccezioni, rappresentate perlopiù dai membri del Rotary Club del luogo. Keys lanciò il Seven Countries Study of Cardiovascular Diseases rilevando l'importanza della dieta mediterranea e documentandone i benefici sia sulle patologie croniche cardiovascolari sia sul miglioramento dello stato di salute nel tempo. L'esame di 12000 soggetti tra i 40 ed i 60 anni, residenti in diversi paesi come il Giappone, gli USA, I'Olanda, la Jugoslavia, la Finlandia e I'Italia, confermò l'ipotesi di Keys: quanto più l'alimentazione dei soggetti esaminati si allontanava dagli schemi mediterranei, maggiore era l'incidenza delle cosiddette "malattie del benessere" [8]

Terminate le sue ricerche, Keys scrisse che l'essenza della mediterraneità della dieta risultava essere fondamentalmente vegetariana: pasta e cereali in varie forme, verdure condite con olio di oliva e ogni sorta di frutta e ortaggi di stagione. Da allora si sono susseguiti molti altri studi che hanno meglio documentato e definito l'efficacia di essa in varie patologie, indagando a fondo sulle sue proprietà nutritive. La letteratura scientifica è molto ampia e corposa a riguardo.

II Progetto SUN [9] dell'Università di Navarra in Spagna, ad esempio, ha studiato l'effetto della dieta sull'ipertensione, sul diabete, sulle dislipidemie, sull'obesità, sulla malattia coronarica e su altre patologie con circa 18000 soggetti reclutati. I risultati disponibili fino ad ora rilevano che vi sarebbe un'associazione inversa tra l'olio extravergine d'oliva o tra l'adesione a un modello alimentare mediterraneo e l'infarto del miocardio; in particolare per gli uomini, dopo 28 mesi di dieta mediterranea ricca di olio d'oliva si evidenziava un ridotto rischio d'ipertensione ed un abbassamento della colesterolemia, suggerendo dunque la superiore qualità dei grassi assunti tramite la dieta mediterranea come possibile causa di questi effetti benefici.

Lo Studio ATTICA [3], condotto negli anni 2001-2002, cui hanno partecipato 3024 soggetti (di età compresa fra 20 e 89 anni, per la maggior parte uomini della regione greca di Attica), ha dimostrato che una maggiore aderenza riduce il rischio per lo sviluppo della disfunzione sistolica ventricolare sinistra nei pazienti affetti da sindrome coronarica. Lo Studio EPIC (European Prospective Investigation into Cancer and Nutrition) [10] è il più vasto studio di popolazione condotto sui rapporti tra dieta e salute, a cui hanno preso parte 520000 persone provenienti da dieci paesi europei. Lo studio EPIC tentò di chiarire i rapporti tra dieta, fattori ambientali, stile di vita e incidenza di cancro e di altre malattie croniche. Ha evidenziato come un incremento dell'aderenza alla dieta mediterranea corrispondesse a una riduzione dell'incidenza di cancro.

II Lyon Diet Heart Study [11] ha accertato che una dieta di tipo mediterraneo può diminuire il tasso di recidiva dopo un primo infarto miocardico. I risultati dello studio, di tipo randomizzato, mostrano che l'effetto protettivo della Dieta Mediterranea si mantiene fino a quattro anni dopo l'infarto.

Lo Studio GISSI-Prevenzione [12] ha invece dimostrato come nei pazienti con infarto miocardico che sono riusciti a mettere in pratica alcuni consigli dietetici, in particolare con l'aumento del consumo di cibi mediterranei, il rischio di morte precoce sia diminuito, indipendentemente da qualsiasi trattamento farmaceutico.

\section{il. Assetto Lipidico e Rischio Cardiovascolare: Possibile Ruolo Della Nutrizione}

Tra i parametri di rischio cardiovascolare la dislipidemia è un fattore rilevante e indipendente ma ha il vantaggio di essere modificabile. Nelle linee guida sul trattamento delle dislipidemie ESC/EAS [13] si sottolinea l'importanza di un'adeguata dietoterapia per raggiungere desiderabili valori di colesterolemia e trigliceridi, come componente fondamentali delle strategie attuate da società scientifiche internazionali per prevenire dislipidemie e cardiovasculopatie. Gli obiettivi sono quelli di attuare una sana alimentazione per raggiungere un peso corporeo ideale, attenersi ai livelli raccomandati di lipoproteine a bassa densità LDL, ad alta densità HDL, trigliceridi, zuccheri semplici, praticare attività fisica e controllare la pressione arteriosa.

Nel corso degli anni numerosi studi epidemiologici e fisiopatologici hanno messo in luce che dislipidemie e obesità viscerale sono strettamente connesse tra loro [14].

La quantità di tessuto adiposo addominale, stimata con la misurazione della circonferenza vita, è direttamente proporzionale all'insulino-resistenza, all'aumento della concentrazione plasmatica di trigliceridi (ipertrigliceridemia) e LDL e ad un prolungato tempo di circolo nel plasma di queste lipoproteine e dei loro prodotti catabolici, altamente aterogeni [15].

Una correlazione inversa tra livelli di colesterolo HDL e rischio cardiovascolare è ampiamente documentata in letteratura. L'analisi combinata di quattro studi prospettici americani, ha messo in luce come per ogni decremento di $1 \mathrm{mg}$ di HDL il rischio cardiovascolare aumentava del 3\% nelle donne e del $2 \%$ negli uomini [16].

Diversi effetti ateroprotettivi sono stati attribuiti alle HDL e dimostrati negli ultimi 20 anni. Tra i più importanti la protezione dalla perossidazione delle LDL 
e dai danni cellulari provocate dalle stesse LDL ossidate; il ruolo centrale delle HDL nel trasporto inverso del colesterolo dai tessuti periferici al fegato; gli effetti antinfiammatori sui monociti e sulla componente cellulare delle pareti vasali; l'aumento della vasodilatazione arteriosa; l'inibizione dell'aggregazione piastrinica; l'inibizione del reclutamento di cellule infiammatorie all'interno della parete arteriosa [17-19].

L'accumulo di grasso viscerale rappresenta dunque un fattore di rischio di malattia cardiovascolare. La dislipidemia che tipicamente si associa all'obesità viscerale è caratterizzata da tre fattori: aumento della concentrazione plasmatica di trigliceridi, presenza di lipoproteine a bassa densità in prevalenza (più piccole e dense del normale marcatamente aterogene) e bassi livelli di colesterolo nelle lipoproteine ad elevata densità. Tale fenotipo lipidico è soventemente associato a placche ateromasiche instabili e ad un marcato aumento dei markers di infiammazione. Dato lo stretto legame tra grasso viscerale e alterazione dell'assetto lipidico, appare clinicamente utile per la correzione della dislipidemia, l'introduzione di appropriate norme dietetiche e dell'incremento dell'attività fisica, come raccomandato dalle linee guida dell'American Diabetes Association e del NCEP-ATP III $[11,20]$.

La dislipidemia aterogena rappresenta uno dei casi in cui il pattern dietetico mediterraneo esplica il suo effetto protettivo modulando favorevolmente alcuni importanti fattori di rischio.

Studi epidemiologici [8] hanno osservato una grande differenza geografica nei tassi di incidenza di malattie cardiovascolari. Rispetto ai paesi del nord Europa o negli Stati Uniti, nei paesi del sud Europa, come la Francia, la Spagna, in Grecia e in Italia vi è una bassa incidenza di malattia coronarica (CHD). II modello alimentare mediterraneo è stato il fattore più frequentemente invocato per spiegare questa differenza.

La prima piramide alimentare mediterranea fu elaborata da Willet nel 1993: essa prevedeva un consumo giornaliero abbondante di cereali, patate, frutta fresca e secca, vegetali, legumi, olio d'oliva e piccolo o moderato consumo di formaggio, e yogurt; poche volte a settimana piccole o moderate quantità di pesce, pollame, uova e dolci (zuccheri concentrati, miele); poche volte durante il mese piccole quantità di carne rossa. II vino era ammesso con moderazione e veniva consigliata un'attività fisica abituale. La revisione 2009 delle Linee guida redatta dall'Istituto Nazionale di Ricerca per gli Alimenti e la Nutrizione (INRAN), presenta la nuova piramide alimentare, la stessa utilizzata nel corso di tale studio. La nuova piramide (Fig.1) sostituisce la precedente e si rivolge alla popolazione italiana di età compresa tra i 18 e i 65 anni. Rispetto alla piramide precedente, non dà indicazioni iconografiche degli alimenti ma, fornisce indicazioni sulla frequenza di consumo giornaliera e settimanale.
Tuttavia, è difficile definire la dieta mediterranea come una caratteristica comune di tutti i paesi del Mediterraneo, poiché esistono differenze notevoli. Ad esempio, la variante italiana della dieta mediterranea è caratterizzata da un maggior consumo di pasta, mentre in Spagna, il consumo di pesce è particolarmente elevato. In Grecia, si consumano grandi quantità di pane integrale, cibi cotti e insalate ricche di olio d'oliva, dove verdure e legumi sono compaiono in grandi quantità. Tuttavia, in tutti i casi nell'alimentazione del bacino mediterraneo il rapporto grassi monoinsaturi/ saturi è molto superiore rispetto al nord Europa e Nord America [21] così come l'apporto di molecole antiossidanti e antinfiammatorie, per il più abbondante consumo sia di frutta e verdure fresche [22-26], sia di olio extravergine di oliva come condimento [27], sia per il maggior consumo di prodotti ittici e dunque di PUFAs [28] nonché di altre sostanze bioattive di origine marina [29-34].

\section{ili. Materiali e Metodi}

Al fine di dimostrare come la dieta mediterranea possa avere effetti benefici sulla salute umana rispetto a un modello dietetico tipicamente occidentale tendenzialmente iperproteico, abbassando i livelli di colesterolo e trigliceridi e quindi il rischio di sviluppare patologie cardiovascolari e diabete di tipo 2, abbiamo testato la sua efficacia su un campione di adulti abruzzesi non affetto da dislipidemia su base familiare senza alcuna evidenza clinica di CHD. II trial prospettico sperimentale è stato svolto presso l'Unità Operativa di Nutrizione Umana e Clinica del Dipartimento di Scienze Orali Mediche e Biotecnologiche dell'Università di Chieti, con l'obiettivo di testare significativamente l'efficacia della dieta mediterranea su pazienti con alterazioni dell'assetto lipidico, utilizzando la piramide alimentare come unico strumento terapeutico per tutto l'iter di indagine, in assenza di qualsiasi tipo di terapia farmacologica coadiuvante. Nel trial clinico sono stati adottati sul campione arruolato due modelli alimentari diversi, in corrispondenza dei quali abbiamo registrato cambiamenti significativi dei livelli sierici di lipoproteine e trigliceridi. Abbiamo osservato le associazioni di questi due modelli alimentari con il rischio di sviluppare malattie cardiovascolari monitorando l'andamento dei biomarkers (colesterolo totale, LDL, HDL, trigliceridi) e le variazioni del WHR e del BMI. Per evitare che le disuguaglianze riscontrate tra i due gruppi sui livelli di Colesterolo LDL o HDL indotte dalle due diete potessero essere associate a differenze biologiche di sesso e di età, sono stati inseriti nello studio sia uomini che donne, di età compresa tra i 35 e i 45 anni e l'aderenza dei pazienti ai modelli dietetici somministrati è stata rigorosamente e periodicamente controllata. Sono stati arruolati 40 soggetti, 20 uomini e 20 donne di professioni diverse e di età compresa tra i 35 e i 40 anni, 


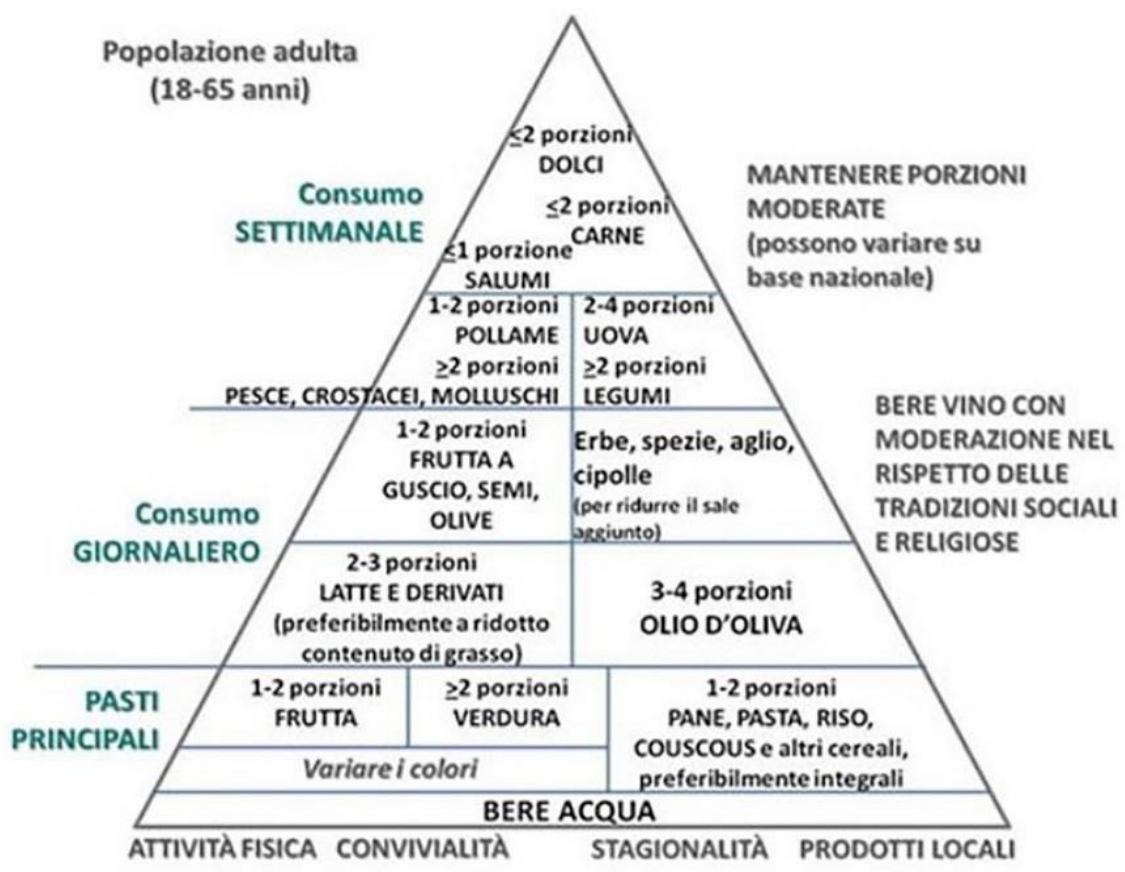

Figura 1: La nuova piramide alimentare della dieta mediterranea proposta dalle Linee Guida INRAN 2009

suddivisi, in maniera randomizzata, in due gruppi da 20 , contenente ciascuno 10 uomini e 10 donne. Per il reclutamento del campione i partecipanti al trial non potevano avere abitudini tabagiche (almeno un anno e mezzo di astinenza dal fumo), né alcun trattamento farmacologico in corso; altri criteri di esclusione sono stati l'assunzione di integratori o pillola anticoncezionale e pregresse diagnosi di infarto cardiaco, angina pectoris, diabete, ulcere duodenali o gastriche, ictus cerebri, patologie epatiche, calcoli alla cistifellea. Tutti presentavano assetto lipidico alterato secondo le Lineeguida del National Cholesterol Education Program (NCEP) con livelli di Colesterolo Totale $>200 \mathrm{mg} / \mathrm{dl}$, $\mathrm{HDL}<45 \mathrm{mg} / \mathrm{dl}, \mathrm{LDL}>130 \mathrm{mg} / \mathrm{dl}$ e Trigliceridi $>150$ $\mathrm{mg} / \mathrm{dl}$ [35]. Ogni partecipante è stato sottoposto inizialmente ad anamnesi familiare, clinica e patologica, a valutazione della composizione corporea (peso, altezza, circonferenza vitae, circonferenza fianchi, WHR, $\mathrm{BMI})$, dello stato nutrizionale e a un recall dietetico delle 24 ore, utile per conoscere le abitudini alimentari e l'apporto idrico medio quotidiano. Da ogni referto clinico, sono stati estrapolati i livelli di Colesterolo totale, LDL, HDL e Trigliceridi. I pazienti sono stati classificati secondo la World Health Organization BMI Classification [36] in: sottopeso (BMI <18.4), normopeso (BMI 18.524.99), sovrappeso (BMI 25.0-29.9), obesità grado I (30.0-34.9), obesità grado II (BMI 35.0-39.9), obesità grado III (BMI > 40) e in base al WHR in obesità ginoide, androide o mista. Per ciascuno è stato calcolato il metabolismo basale $(\mathrm{MB}) \mathrm{e}$ il relativo fabbisogno calorico giornaliero.
II modello alimentare mediterraneo (Dieta $\mathrm{M}$ ) impiegato per il primo gruppo, è stato elaborato secondo le linee guida redatte dall'INRAN, facendo riferimento all'ultima revisione del 2009 [37]. Dopo aver calcolato il Metabolismo Basale tramite la formula di Harris-Benedict [38] e il fabbisogno energetico giornaliero totale, tenendo conto del livello di attività fisica svolta (LAF), sono state formulate diete personalizzate rispettando porzioni settimanali suggerite delle Linee Guida INRAN, adattando le grammature all'introito calorico necessario (Fig.2). Si definisce porzione la quantità standard di alimento espressa in grammi, che si assume come unità di misura da utilizzare in un'alimentazione corretta al fine di soddisfare il fabbisogno nutrizionale del consumatore. 


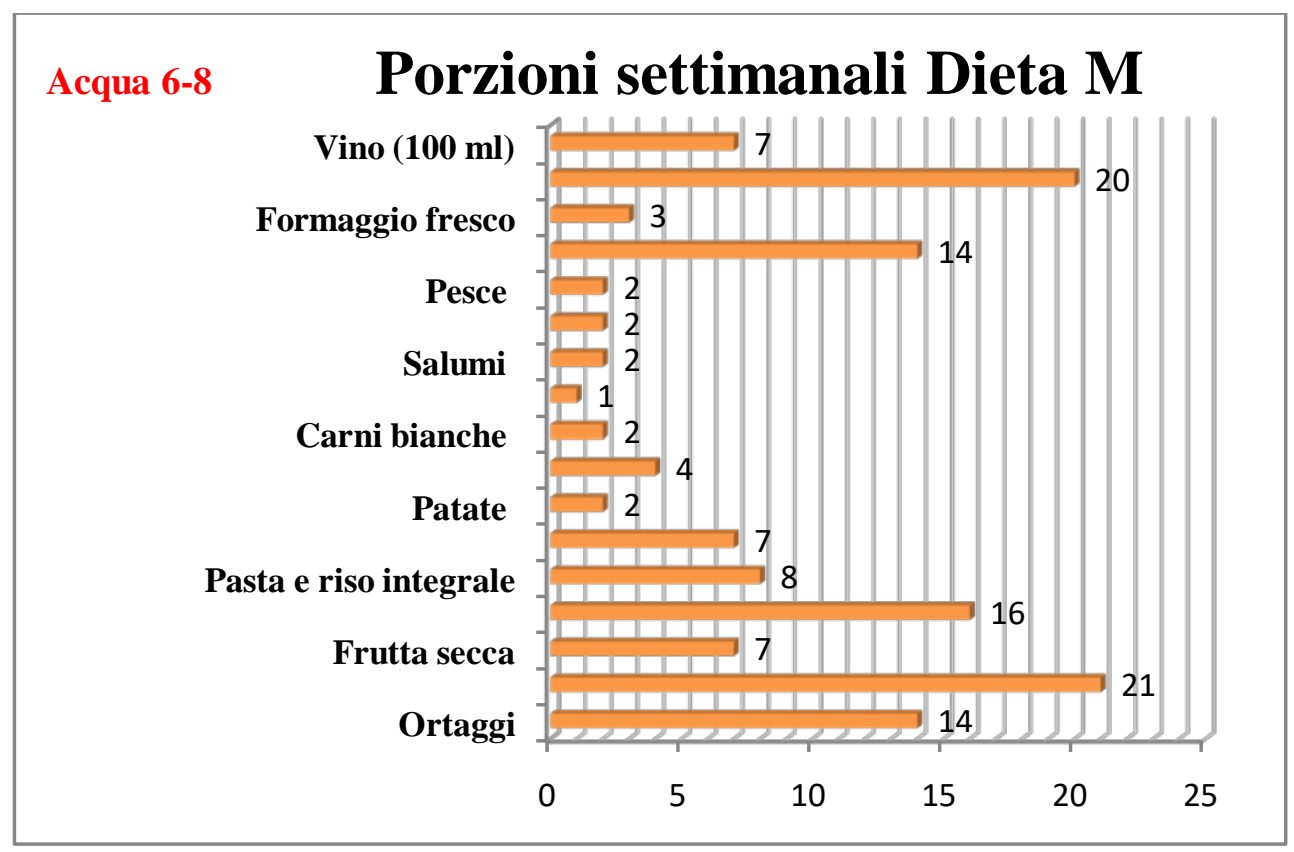

Figura 2: Porzioni settimanali suggerite nella dieta M, secondo le Linee Guida INRAN del 2009. ripartito:

Il fabbisogno calorico giornaliero è stato così

- Carboidrati 55-57\% delle kcal tot/die (pane, pasta e riso integrali, miglio, orzo, farro, quinoa, bulgur, avena, legumi, ecc.)

- Lipidi 26-28\% delle kcal tot/die (olio extravergine d'oliva) così ripartiti: grassi saturi non più del 7-10\% delle kcal totali (burro, margarina, pancetta, crema di nocciole e cacao, carne rossa, formaggi stagionati); acidi grassi monoinsaturi fino al $20 \%$ delle kcal totali (olio extravergine d' oliva), acidi grassi polinsaturi circa il $7 \%$ delle kcal totali con un rapporto omega-6/omega-3 intorno a 5:1 ( $\omega 6$ : noci, cereali, pane integrale, oli vegetali; $\omega 3$ : pesce (EPA e DHA), noci (ALA), oli vegetali come l'olio di lino)

- Proteine $15-17 \%$ delle kcal tot./die (proteine animali: carne bianca, uova, pesce, latte e derivati, proteine vegetali: cereali, legumi, frutta secca.)

- Il consumo di sale è stato limitato a $5 \mathrm{~g}$ al giorno, pertanto il sodio a $2.5 \mathrm{~g} / \mathrm{die}$.

- Zuccheri semplici non più del $10 \%$ delle kcal totali

- Fibra 30g/die (preferibilmente solubile)

- Frutta secca oleosa intorno ai $20 \mathrm{~g} / \mathrm{die}$

- 5 porzioni/die di frutta e verdura

- Acqua 1.5-2 I/die

- I sei pasti totali giornalieri prevedevano la seguente ripartizione calorica:

- Colazione $20 \%$ delle kcal tot.

- Spuntino $15 \%$ delle kcal tot.

- Pranzo $40 \%$ delle kcal tot.

- Merenda $5 \%$ delle kcal tot.

- Cena $30 \%$ delle kcal tot.

Dopocena: infusi, thè, tisane, camomilla senza
È stata inoltre consigliata un'attività fisica leggera di circa 30 minuti/die (per es. passeggiata).

Il pattern dietetico del gruppo controllo (dieta L), di stampo occidentale, pur essendo sempre ipocalorico, differiva una maggiore assunzione di carne rossa e di prodotti lattiero-caseari, cereali raffinati e ridotto consumo di frutta e verdura. (Fig. 2 e 3).

Il fabbisogno calorico giornaliero è stato così di seguito ripartito:

- Carboidrati $(\mathrm{CHO})$ intorno al 30\% delle kcal tot/die (pasta e pane non integrali e cereali perlopiù raffinati)

- Proteine $30 \%$ (proteine animali > proteine vegetali)

- Lipidi $40 \%$ (acidi grassi saturi $>10 \%$, monoinsaturi $<20 \%, \omega 6>\omega 3)$

- Nessuna indicazione specifica per il consumo di frutta fresca e secca, verdure, zuccheri semplici, fibra, sale, acqua e attività fisica.

II follow-up è stato stabilito per sei mesi con un primo controllo dei parametri antropometrici, ematochimici e nutrizionali a distanza di tre mesi circa. Durante tutto il periodo di studio, ogni paziente è stato sollecitato a manifestare le proprie impressioni, sensazioni, e difficoltà riscontrate durante l'attuazione dei pattern dietetici. Inoltre nessun paziente ha abbandonato il trattamento o è rientrato nel drop-out per scarsa compliance o per averlo modificato senza previa consultazione. zucchero 


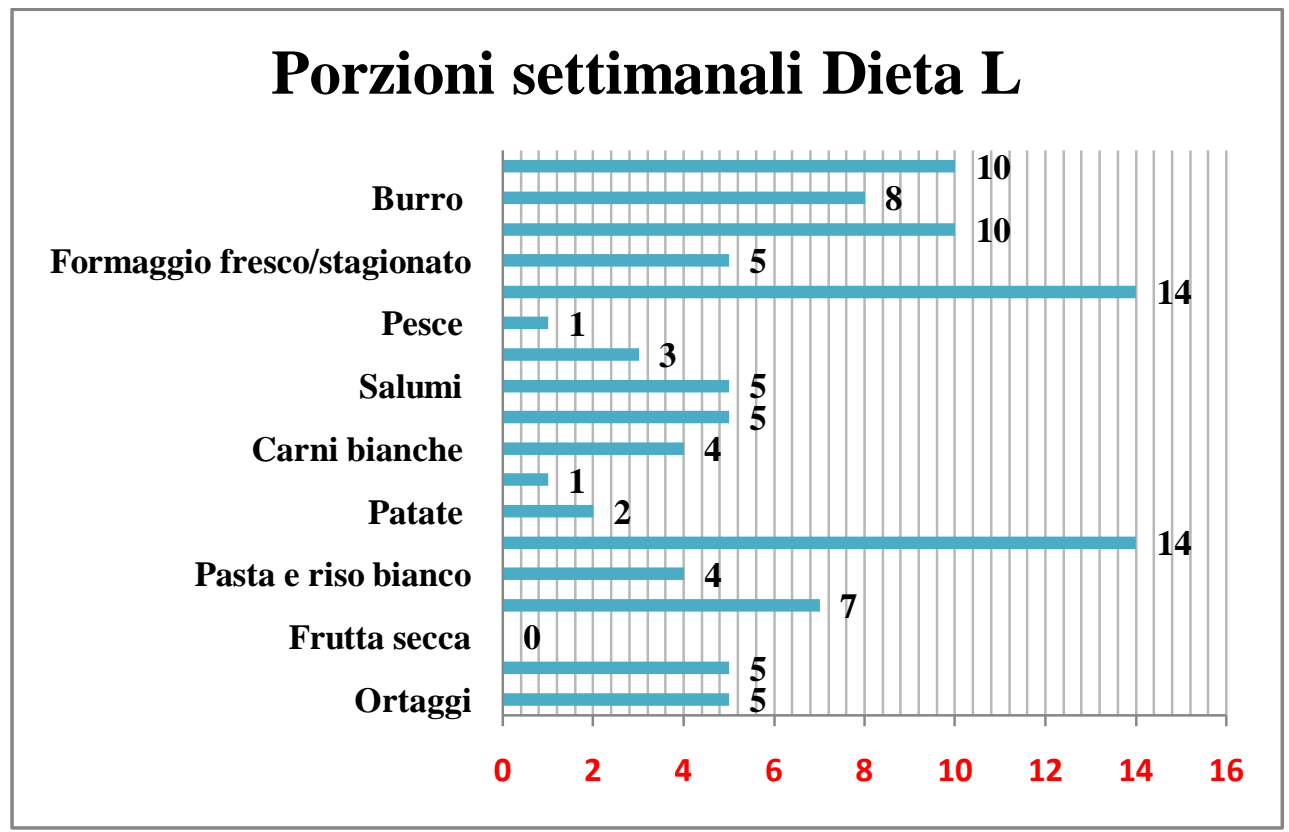

Figura 3a: Porzioni settimanali assunte dal gruppo in dieta L

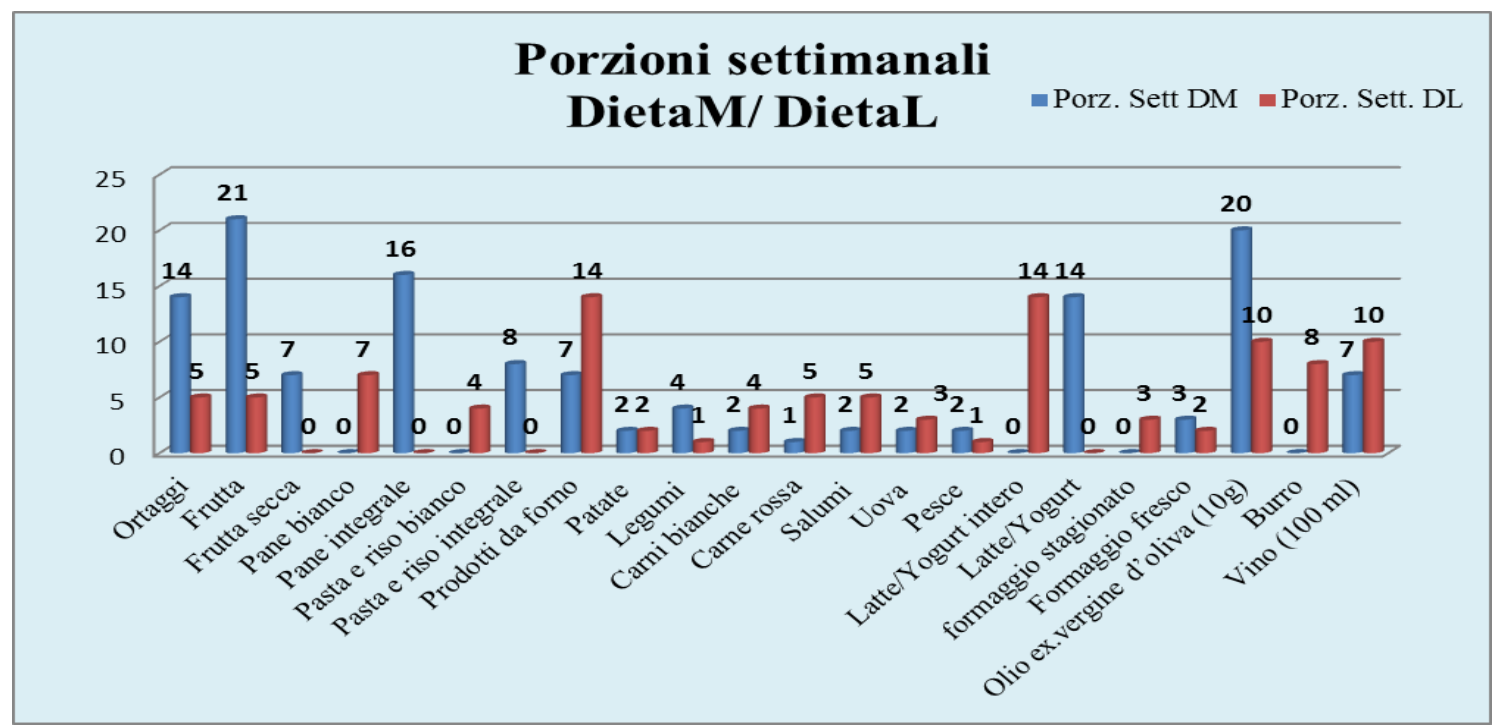

Figura 3b: Confronto tra le frequenze settimanali di assunzione delle principali categorie di alimenti nelle due diete messe a confronto.

\section{Risultati}

I 40 soggetti arruolati nello studio non avevano ricevuto nessuna indicazione dietetica prima del trial. Sono stati seguiti per circa sei mesi. Dopo la prima fase di assessment, a distanza di tre mesi, è stato effettuato uno screening ematologico e tutti i partecipanti sono stati rivalutati da un punto di vista antropometrico e nutrizionale. Le variabili monitorate in ciascun gruppo con i due diversi pattern dietetici sono state: peso, circonferenza vita, WHR, BMI, colesterolo totale, LDL, HDL e trigliceridi. L'assetto lipidico dei soggetti in dieta $\mathrm{M}$ ha dimostrato una miglior risposta, mentre il gruppo in dieta $L$ ha subito un graduale peggioramento dei livelli ematici di LDL e HDL nonostante un lieve calo ponderale dei soggetti con BMI > 24.9. Per ciascuna variabile considerata è stata fatta distinzione dei sessi all'interno dei due gruppi di studio.

Al primo controllo, a 3 mesi, i partecipanti di sesso maschile e femminile in dieta $\mathrm{M}$ hanno mostrato una variazione percentuale media $(\overline{\mathrm{M}} \Delta \%)$ della circonferenza vita rispettivamente del -2 \% e del -2.86\% circa rispetto ai valori iniziali, mentre per i partecipanti di sesso maschile e femminile in dieta $L$ questa variazione risultava rispettivamente del $-1.6 \%$ e del $-0.94 \%$ circa. Anche il peso corporeo ha subito una riduzione del $4.8 \%$ circa negli uomini e del $-3.5 \%$ nelle donne in dieta $\mathrm{M}$; del $-2.1 \%$ negli uomini e del $-1.2 \%$ nelle donne in dieta $\mathrm{L}$. 
Al secondo controllo, a 6 mesi la $\overline{\mathrm{M}} \Delta \%$ della circonferenza vita rispetto al primo è stata la seguente: circonferenza vita per le donne $-2.80 \%$ e per gli uomini $3.68 \%$ in dieta $\mathrm{M}$; per le donne $-0.74 \%$ e per gli uomini $1.13 \%$ in dieta $L(p<0.05)$.

A fine studio è stata calcolata la $\bar{M} \Delta \%$ della circonferenza vita rispetto alla fase iniziale (dopo 6 mesi): il gruppo in dieta $\mathrm{M}$ ha subito complessivamente una riduzione del $-7.99 \%$ ( $p<0.05)$, mentre il gruppo in dieta $L$ del $-5 \% \quad(p<0.05)$. Mentre la variazione percentuale del peso è stata $-7.77 \%(p<0.05)$ nel gruppo DM essa è stata pari a $-3.2 \%(p<0.05)$ del gruppo DL.

Tali variazioni sono state poi confermate dal calcolo del BMl che, al termine del follow-up dopo i 6 mesi, ha mostrato nelle donne un decremento medio percentuale $(p<0.01)$ del $-18.5 \%$ in dieta $\mathrm{M}$ e del $-7.6 \%$ nelle donne in dieta $L$ (Fig. 4a). Ancor più positivi sono stati i risultati dei partecipanti di sesso maschile in dieta $\mathrm{M}$ con una variazione percentuale $(\mathrm{p}<0.01)$ del BMI del $-24.5 \%$, rispetto al $-10.4 \%$ degli uomini in dieta $L$ (Fig. 4b). Dopo il primo e il secondo trimestre di intervento dietetico sono state valutate le variazioni nell'assetto lipidico. Trascorsi sei mesi dalla fase iniziale, confrontando i valori registrati al primo controllo con quelli raccolti al secondo, si nota che il valore medio del colesterolo totale del gruppo in dieta $\mathrm{M}$ è inferiore a quello del gruppo in dieta $L$ (fig. $5 a$ ). Dai valori registrati al secondo controllo (a sei mesi) si nota che il valore medio del colesterolo LDL del gruppo in dieta $M$ è inferiore rispetto al valore medio del gruppo in dieta $L$ (fig. 5b). A sei mesi (ossia al secondo controllo), abbiamo riscontrato una differenza significativa anche a carico del colesterolo HDL (fig. 5c) e dei trigliceridi (fig. 5d) con i risultati migliori sempre per il gruppo in dieta M.

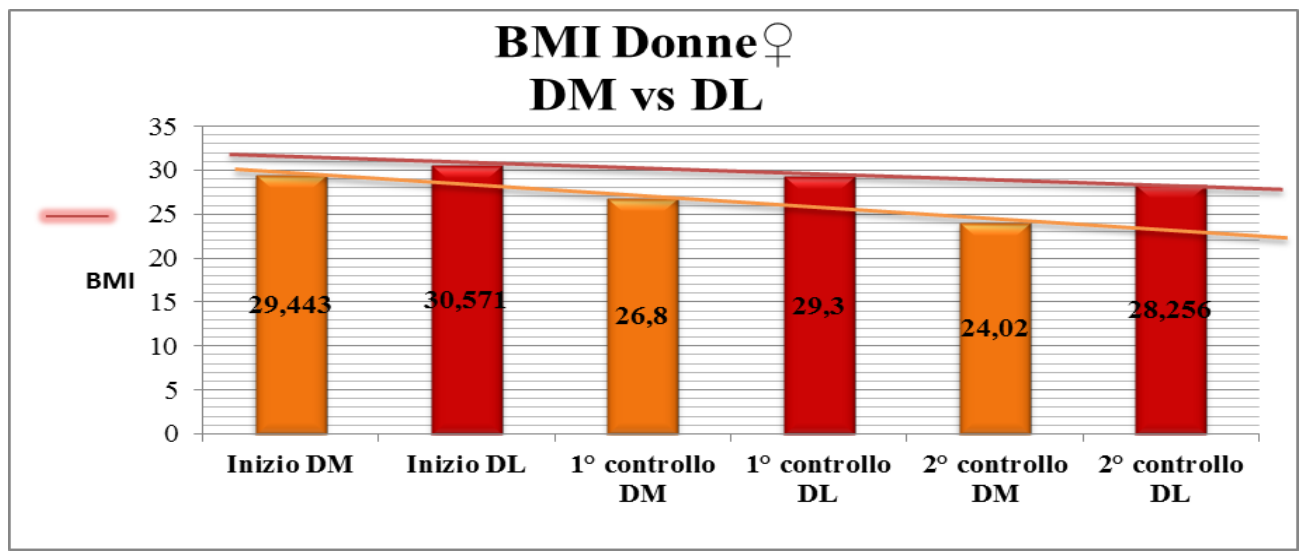

Figura 4a: Variazione percentuale media del BMI nelle donne in dieta M confrontata a quella delle donne in dieta $\mathrm{L}$ dall'inizio dello studio al primo (-9\% in DM rispetto al $-4.3 \%$ in $\mathrm{DL})$ e secondo controllo $(-10.4 \%$ in DM rispetto a $3.6 \%$ in $\mathrm{DL})$.

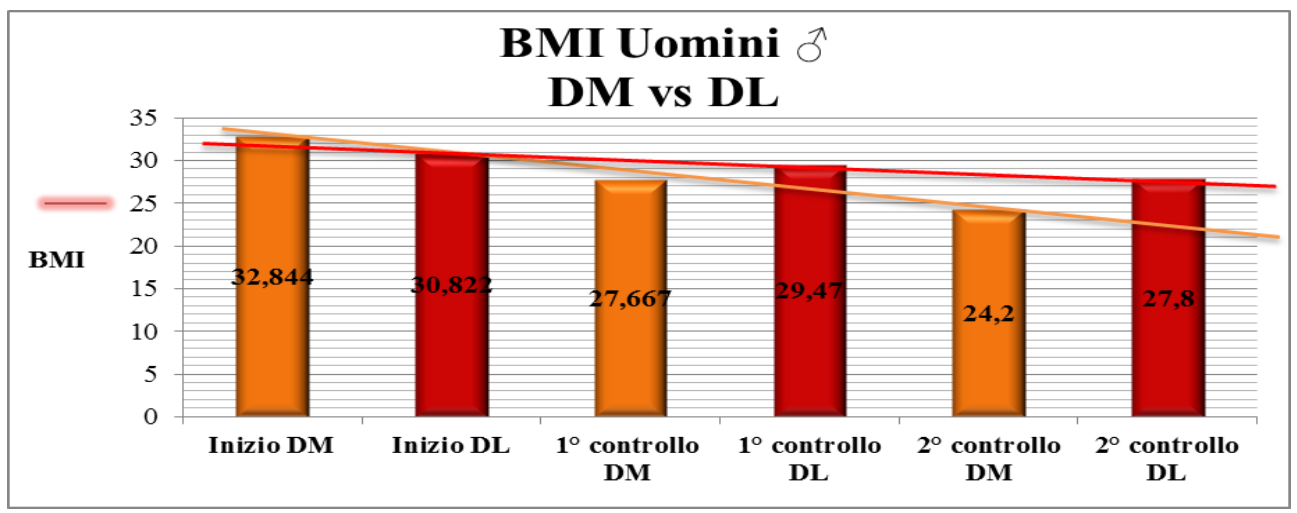

Figura 4b: Variazione percentuale media del BMI nelle donne in dieta $\mathrm{M}$ confrontata a quella delle donne in dieta $\mathrm{L}$ dall'inizio dello studio al primo (-12.7\% in DM rispetto al $-4.4 \%$ in $\mathrm{DL})$ e secondo controllo (-11.73\% in DM rispetto a $5.6 \%$ in $\mathrm{DL})$. 


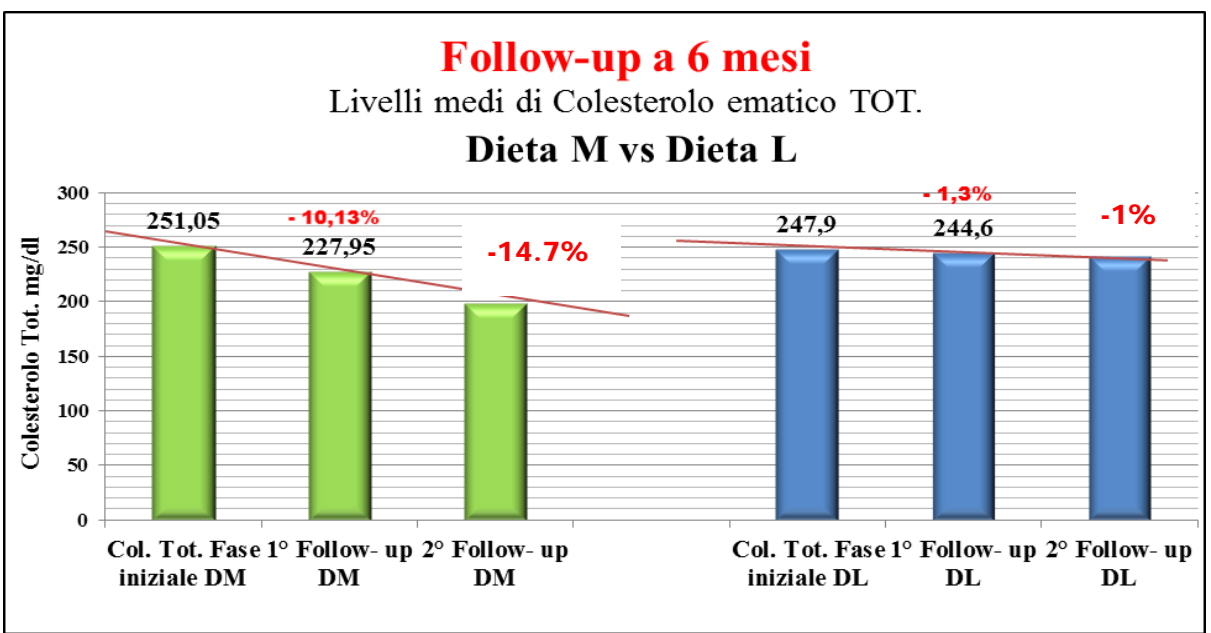

Figura 5a: Variazione dei livelli di Colesterolo totale nei due gruppi di pazienti tra la fase iniziale, il primo ed il secondo controllo.

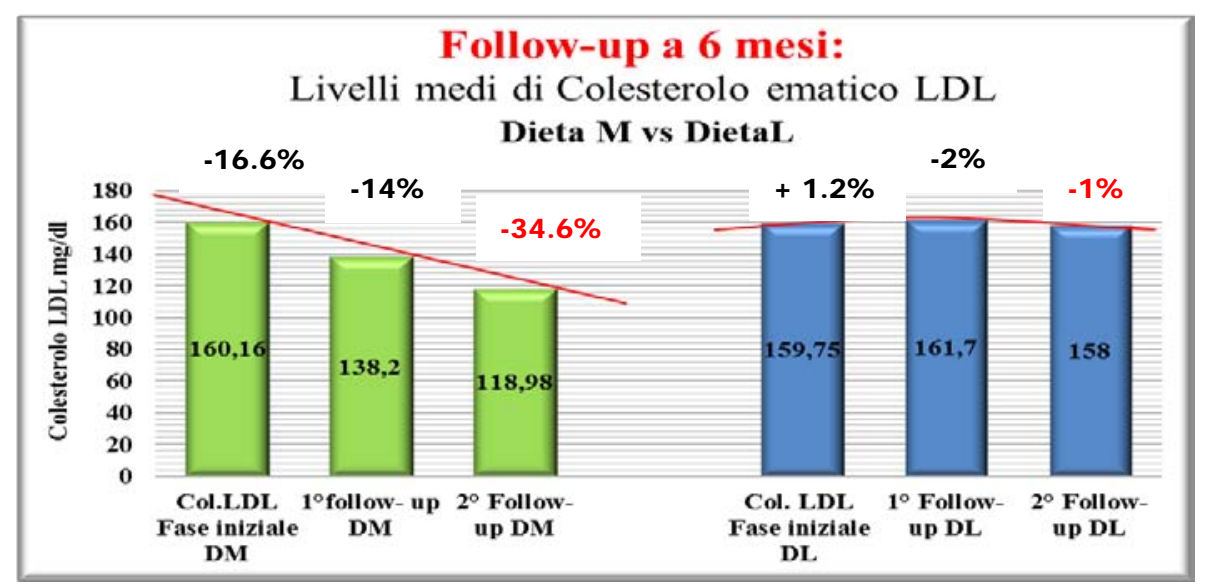

Figura 5b: Variazione dei livelli di Colesterolo LDL nei due gruppi di pazienti tra la fase iniziale, il primo ed il secondo controllo.

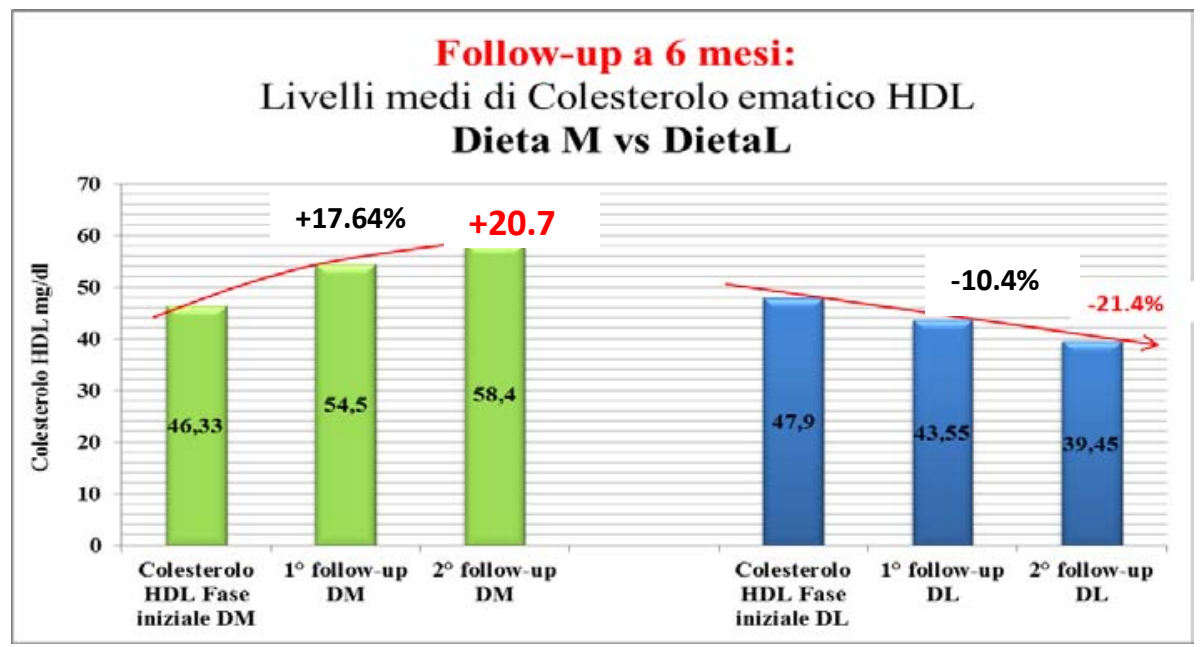

Figura 5c: Variazione dei livelli di Colesterolo HDL nei due gruppi di pazienti tra la fase iniziale, il primo ed il secondo controllo. 


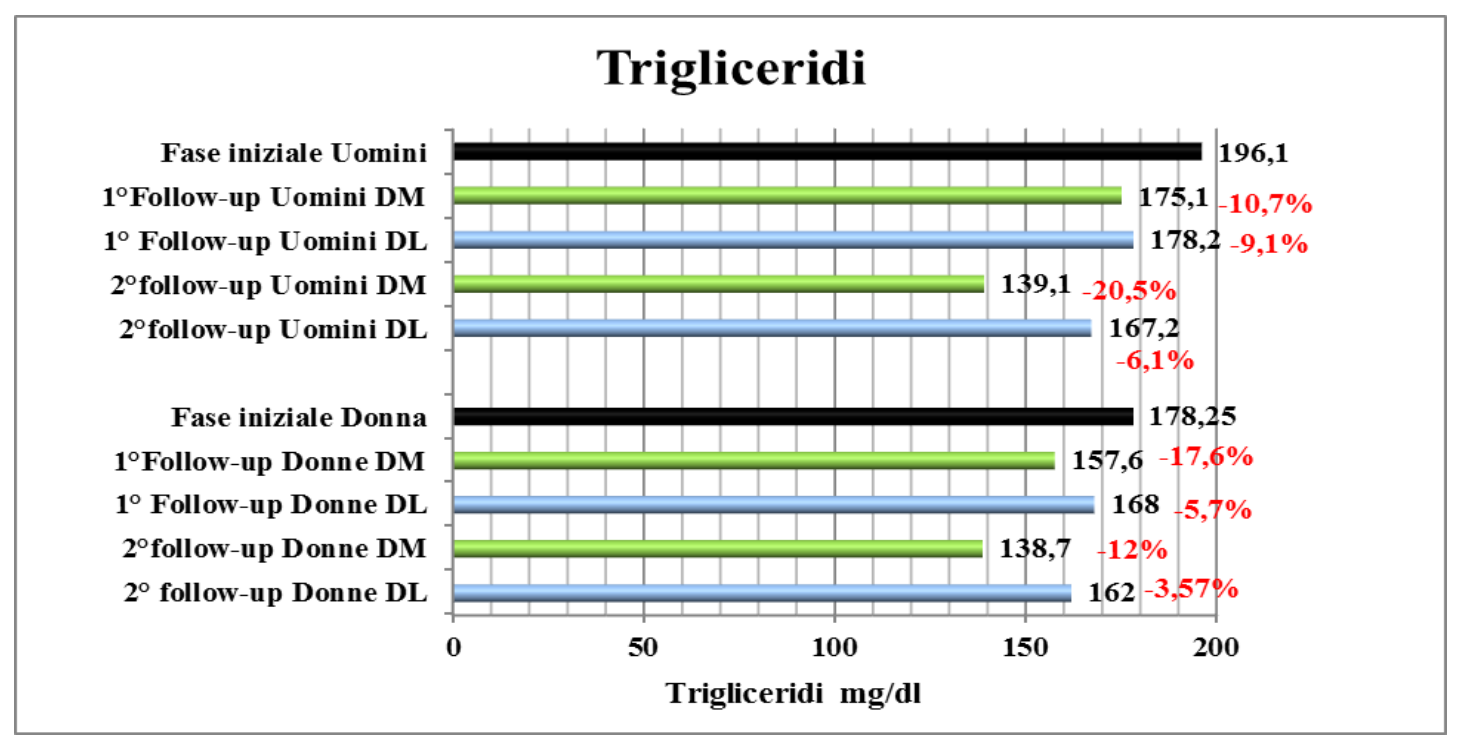

Figura 5d: Variazione dei livelli di trigliceridi nei due gruppi di pazienti tra la fase iniziale, il primo ed il secondo controllo con distinzione tra i sessi.

\section{Discussione}

I partecipanti in DM hanno consumato, secondo il pattern dietetico somministrato, più verdura, più frutta di stagione, frutta oleosa, olio extra vergine d'oliva, più pesce e cereali integrali rispetto al gruppo DL. Tali partecipanti invece hanno seguito un pattern dietetico pur ipocalorico ma senza indicazioni di frequenza di consumo ad indirizzo "mediterraneo", pertanto consumando per sei mesi più carne rossa, cereali raffinati, meno olio extravergine d'oliva, più prodotti lattiero caseari freschi e stagionati, più affettati e meno vegetali. Entrambi i gruppi hanno incrementato l'attività fisica con una camminata di 30 minuti al giorno, praticata 3 volte a settimana.

Al termine del trial abbiamo osservato associazioni significative tra il modello dietetico mediterraneo e quello occidentale con diversi biomarkers plasmatici.

In particolar modo la dieta $L$ è risultata significativamente $(p<0.001)$ correlata ad un peggioramento del colesterolo HDL e LDL per entrambi i sessi (HDL gruppo DM: +20.7\%; HDL gruppo DL: -21.4\%; LDL gruppo DM: -34.6\%; LDL gruppo DL: -1\%).

Inoltre anche il WHR nel gruppo DL non ha mostrato importanti cambiamenti, così come il BMl che, dopo sei mesi, pur essendosi ridotto grazie al calo ponderale, non è tuttavia rientrato nel range di normalità: il BMI medio in dieta $L$ è risultato 27.8 (-9.8\%) per il sesso maschile e 28.25 (-7.6\%) per il sesso femminile. II pattern dietetico di stampo mediterraneo ha invece riequilibrato i valori dislipidemici iniziali e migliorato il $\mathrm{BMI}$ che è rientrato nella fascia di normopeso: BMI medio in dieta M 24.2 (-26.3\%) per il sesso maschile e 24.02 (-18.4\%) per il sesso femminile.

Nella valutazione del rischio per la salute umana è importante considerare non solo il BMI, ma anche la distribuzione del tessuto adiposo: è stato, infatti, riconosciuto che un'eccessiva quantità di grasso viscerale è un importante fattore di rischio per la salute, indipendentemente dal BMI. Le persone con un grasso localizzato prevalentemente in sede addominale hanno infatti un aumentato rischio di diabete tipo 2, dislipidemia, ipertensione arteriosa e malattie cardiovascolari [39].

Pertanto al termine dello studio abbiamo calcolato la $\bar{M} \Delta \%(p<0.05)$ della circonferenza vita: nel gruppo in DM è risultata pari a $-4.74 \%$ per il sesso femminile e $-6.41 \%$ per il sesso maschile; nel gruppo in $D L$ è risultata invece pari a $-2.4 \%$ per il sesso femminile e $-1.73 \%$ per il sesso maschile; la $\bar{M} \Delta \%(p<0.05)$ del peso corporeo: nel gruppo in DM $-5.94 \%$ per il sesso femminile e $-9.27 \%$ per il sesso maschile; nel gruppo in DL $-2.6 \%$ per il sesso femminile e $-3.2 \%$ per quello maschile.

Da tali risultati, è ragionevole pensare che la dieta mediterranea, considerata, in base alle sue tradizionali caratteristiche come una dieta prevalentemente ricca in legumi, frutta, verdure, grassi vegetali prevalentemente mono/polinsaturi, è capace di esercitare un benefico effetto sull'adiposità viscerale e sui livelli ematici di colesterolo totale, LDL, HDL e trigliceridi. Durante entrambi i controlli, sono state raccolte informazioni anche sui livelli di sazietà prima e dopo i pasti. Nel gruppo DL il 60\% dei pz, dopo circa un'ora dal pasto, presentava leggera sonnolenza e senso di fame. Mentre nel gruppo DM l'85\% dei pz sentiva senso di sazietà e non presentava sonnolenza. Tutto ciò è giustificato dal fatto che la DM migliora sia la sazietà immediata dopo il pasto che la "satiation" ovvero il senso di fame che precede il pasto successivo. Il tempo di masticazione si allunga così come il tempo di svuotamento gastrico e quindi si modula la 
secrezione di tutti gli ormoni gastroenterici, CCK, grelina, insulina, responsabile della sonnolenza postprandiale. Questo perché la dieta mediterranea ha una bassa densità energetica ed un basso "carico glicemico" grazie al consumo di alimenti preferibilmente integrali a basso indice glicemico.

Anche trigliceridi, colesterolo totale, HDL, LDL dopo sei mesi di trial clinico hanno subito variazioni importanti e significative $(p<0.01)$. La $\bar{M} \Delta \%(p<0.01)$ della trigliceridemia: nel gruppo in DM: $-26 \%$ nel sesso femminile e $-27.8 \%$ nel sesso maschile; nel gruppo in DL - $13.4 \%$ nel sesso femminile e $-10.6 \%$ nel sesso maschile. La $\bar{M} \Delta \%(p<0.01)$ della colesterolemia totale nel gruppo in DM -20.55\% nel sesso femminile e -21.1\% in quello maschile; nel gruppo in $\mathrm{DL}-1.36 \%$ nel sesso femminile e $-0.5 \%$ in quello maschile. La $\bar{M} \Delta \%(p<0.01)$ dell'LDL: nel gruppo in DM $-22.17 \%$ per il sesso femminile e $-30 \%$ nel sesso maschile; nel gruppo in $\mathrm{DL}-2.56 \%$ per il sesso femminile e $+1.22 \%$ per il sesso maschile. La $\bar{M} \triangle \%(p<0.01)$ dell'HDL: nel gruppo in DM $+20 \%$ per il sesso femminile $+28.55 \%$ per quello maschile; nel gruppo in DL $-23.6 \%$ per il sesso femminile e $-14.4 \%$ per il sesso maschile. Le differenze riscontrate sono giustificate dalla diversa percentuale lipidica impostata sul fabbisogno tot/die individuale e dalla scelta degli alimenti. La quota lipidica della DM è in gran parte rappresentata dall'olio extravergine d'oliva con i rispettivi indubbi vantaggi derivanti dalla sua composizione chimica e dall'essere vettore di molte sostanze antiossidanti. II consumo di questo alimento si associa a un basso apporto dietetico di acidi grassi saturi. Le azioni protettive antiaterogene dipendono non solo dal suo elevato contenuto di acidi grassi monoinsaturi (MUFA) ma anche dai suoi componenti fenolici aventi una nota azione protettiva nei confronti dell'ossidazione delle LDL [27]. La dieta può modificare la composizione di quest' ultime: il consumo dell'olio extravergine d'oliva ricco in fenoli aumenta significativamente il contenuto in acido oleico e fenoli delle $L D L$ e le rende più resistenti all'ossidazione, inoltre i fenoli stessi svolgono un'azione cardioprotettiva [23, 24]. I benefici del consumo di olio extravergine d'oliva vanno ben oltre il miglioramento del profilo lipidico e comprendono un effetto antiossidante sulle lipoproteine, antiinfiammatorio vascolare, antitrombotico, di prevenzione della stabilità insulinica, della funzione endoteliale, di miglioramento della pressione arteriosa [27].

Inoltre, l'effetto ipocolesterolemizzante di una dieta ricca in PUFA è riconducibile al binomio acido eicosapentaenoico (EPA) + acido docosaesaenoico (DHA). II consumo di modeste quantità di pesce (2 porzioni a settimana come il pattern DM) che corrispondono a $250 \mathrm{mg} / \mathrm{giorno}$ di apporto di EPA + $\mathrm{DHA}$, riduce il rischio di decesso per $\mathrm{CHD}$ del $36 \%$ in confronto al non consumo [28]. I grassi saturi (SFA) di origine industriale che troviamo in burro, pancetta, agnello, formaggi stagionati, carne rossa ecc. (pattern DL) esplicano un effetto deleterio: aumento del colesterolo LDL, riduzione del colesterolo HDL, aumento dei trigliceridi, promozione dell'insulino-resistenza, alterazione del metabolismo lipidico e bilancio delle prostaglandine in senso pro-trombotico [28], induzione di risposte pro-infiammatorie e di attivazione endoteliale. La frutta secca, inserita solo nel pattern dieta $M$, ha anch'essa influenzato le variazioni percentuali dei livelli sierici di colesterolo LDL e HDL durante il follow-up, come già dimostrato in letteratura scientifica: il suo consumo in piccole quantità è una caratteristica della dieta mediterranea degli anni'60 con una significativa associazione inversa con il rischio di malattia coronarica. II meccanismo attraverso il quale la frutta secca può migliorare lo stress ossidativo con un aumento della resistenza delle LDL all'ossidazione, è rappresentato dall' elevato contenuto in antiossidanti, (fenoli e tocoferoli) localizzati nella pellicola del guscio esterno, PUFA e MUFA.

Gli effetti del consumo di questi alimenti sono stati osservati e analizzati in diversi studi scientifici e risultano essere dose-dipendenti [28].

Al fine di quantificare la capacità che ha un alimento di promuovere la crescita degli ateromi a livello endoteliale in arterie e arteriole, è stato introdotto dal Dipartimento di Nutrizione Clinica e metabolismo lipidico dell'Università dell'Oregon l'indice di aterogenicità [40]. Si calcola per $100 \mathrm{gr}$ di alimento con la seguente formula: $(1.01 \times \mathrm{g}$ ac. grassi saturi $)+(0.05 \times \mathrm{mg}$ colesterolo).

L'aterogenicità di un alimento dipende soprattutto dalla concomitante presenza di elevate quantità di colesterolo ed acidi grassi saturi, ed in particolare dalla concentrazione di questi ultimi. Sono da consumare quelli con indice CSI (Cholesterol Saturated Fat Index) inferiore a 10 (figura 6).

In questo trial per il pattern dieta $\mathrm{M}$, abbiamo seguito le indicazioni favorevoli all'uso di carni bianche (pollame, ovini, suino magro), di pesce. I formaggi sono in gran parte sconsigliati per il loro alto contenuto sia in grassi saturi che in colesterolo. Sono permessi latte, yogurt parzialmente scremati o formaggi scremati. II consumo di uova è stato limitato a 3 uova a settimana.

E bene sottolineare che non tutti i lipidi sono aterogeni, e che alcuni tipi di lipidi possono migliorare il quadro lipidico, come l'olio extravergine d'oliva (fig. 6)

E bene anche ribadire il ruolo protettivo degli antiossidanti nella prevenzione delle malattie degenerative fra cui l'aterosclerosi: le popolazioni con bassa incidenza di coronaropatie ed altre patologie cardiovascolari sono anche quelle in cui è elevato il consumo di frutta, verdura e vino rosso, alimenti che forniscono un elevato apporto di composti ad alto potere antiossidante [22-34]. 

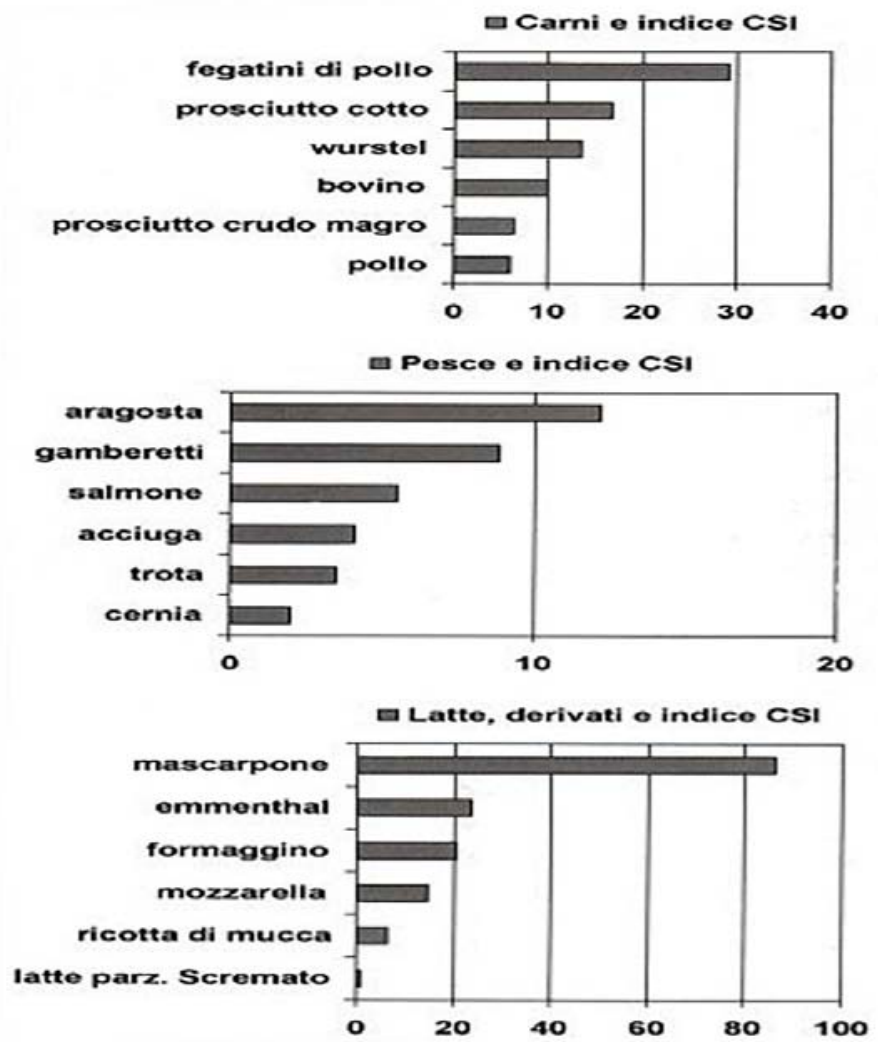

Figura 6: Potenziale ipercolesterolemizzante favorente l'ostruzione di vasi sanguigni INDICE CSI di alcuni alimenti.

Allo schema nutrizionale tipicamente mediterraneo corrisponde un CSI di circa 18 per 1000 $\mathrm{kcal}$, valore da considerare molto buono. La dieta tendenzialmente iperproteica tipicamente occidentale, che prevede il consumo frequente di carne rossa a settimana, insieme ad altri alimenti iperproteici come il parmigiano, insaccati, uova, formaggi stagionati ecc., ha invece un indice CSI molto più elevato.

La dieta italiana è rimasta aderente al modello mediterraneo fino agli anni '60 circa; dopo si è assistito ad un netto allontanamento dal profilo mediterraneo con un consumo di proteine vegetali sempre minore, sempre meno carboidrati, sempre più grassi, in particolare di origine animale. Diversi sono i punti deboli della dieta italiana attuale: troppe proteine animali, troppi grassi (animali e vegetali) a scapito dei carboidrati complessi (fig. 7).

\section{COMPOSIZIONE PERCENTUALE DELL'ENERGIA DISPONIBILE PER IL CONSUMO GIORNALIERO DI ALIMENTI IN ITALIA}

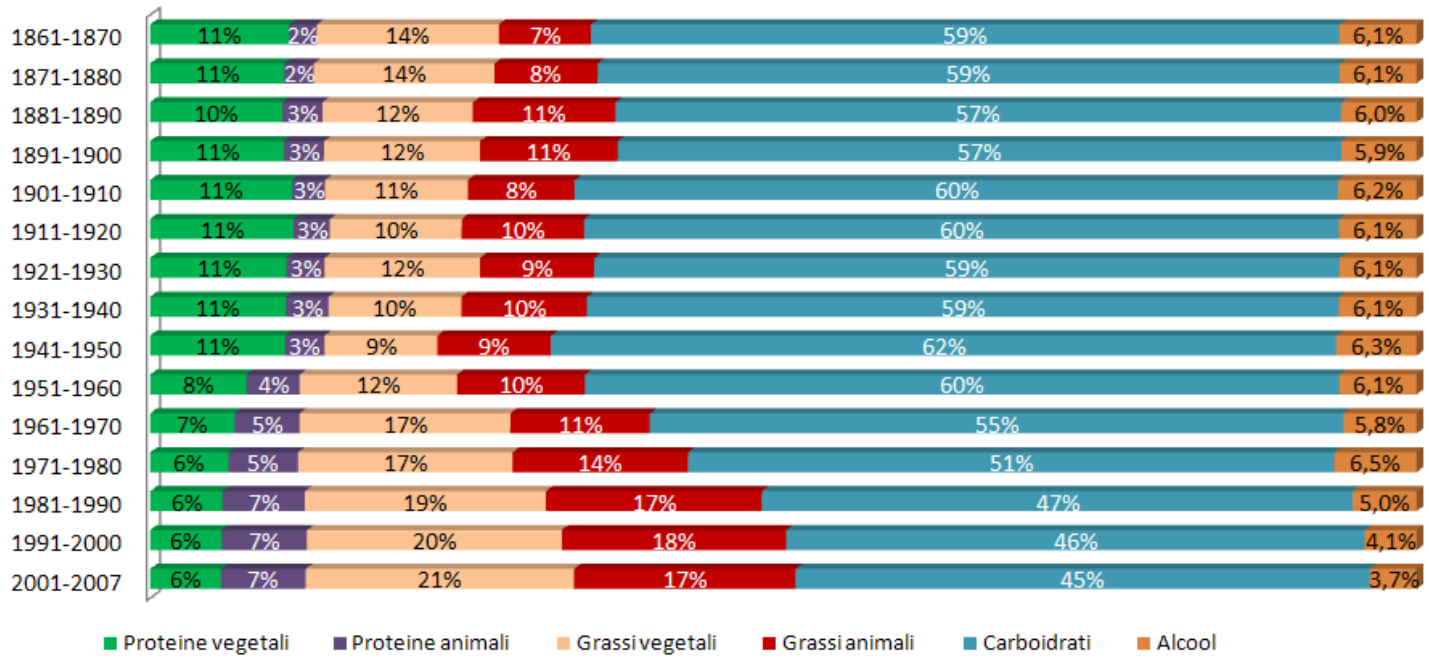

Figura 7: La disponibilità di alimenti in Italia: quanto ci si è allontanati dal modello della Dieta Mediterranea (Elaborazione INRAN su dati ISTAT e FAOSTAT, 2011). 
La dieta mediterranea non è un programma dietetico, ma un vero e proprio stile di vita, con la caratteristica di utilizzare quantità abbondanti di ortaggi, cereali e frutta fresca, l'olio extravergine di oliva come principale fonte di grassi, il consumo frequente di pesce, poca carne e poco vino durante i pasti. Fra tutti i regimi alimentari del mondo, quello mediterraneo ha dimostrato di essere uno dei più sani [41]. Infatti il consumo assiduo di grassi saturi di origine animale all' interno di un regime dietetico prevalentemente proteico, è uno dei fattori di rischio più severi per l'accumulo di grasso viscerale e quindi di malattia cardiovascolare. La dislipidemia che si associa all'obesità viscerale è caratterizzata da: aumento della concentrazione plasmatica di trigliceridi, presenza di lipoproteine a bassa densità in prevalenza più piccole e dense del normale, marcatamente aterogene, bassi livelli di colesterolo nelle lipoproteine ad elevata densità. Tale fenotipo lipidico è sovente associato a placche ateromasiche instabili e ad un marcato aumento dei markers di infiammazione [42], da cui la necessità di instaurare terapie farmacologiche e parafarmacologiche [43] volte alla correzione degli alterati assetti lipidici.

L'alimentazione è uno dei fattori che maggiormente incidono sulla nostra salute in modo diretto e indiretto [44], sullo sviluppo, sul rendimento e sulla produttività delle persone, sulla qualità della vita e sulle condizioni psico-fisiche. Ma soprattutto una dieta corretta può divenire un validissimo e prezioso strumento di prevenzione e di trattamento per numerose patologie.

\section{BiBLIOGRAFIA}

1. Martínez-González M A, Gea A, Ruiz-Canela M. The Mediterranean Diet and Cardiovascular Health. Circ. Res. 2019; 124: 779-798.

2. Jiménez-Cruz A., Jiménez A B, Pichardo-Osuna $A$, et al. Long term effect of Mediterranean diet on weight loss. Nutr. Hosp. 2009; 24(6): 753-754.

3. Tzima N, Pitsavos C, Panagiotakos D B, et al. Mediterranean diet and insulin sensitivity, lipid profile and blood pressure levels, in overweight and obese people; the Attica study. Lipids Health 2007; 6, 22.

4. Pérez-López F R, Chedraui P, Haya J, et al. Effects of the Mediterranean diet on longevity and agerelated morbid conditions. Maturitas 2009; 64(2): 67-79.

5. Rallidis L S, Lekakis J, Kolomvotsou A, et al. Close adherence to a Mediterranean diet improves endothelial function in subjects with abdominal obesity. The American Journal of Clinical Nutrition 2009; 90(2): 263-268.

6. Manuel M, Fernández G, et al. Evidence-based nutritional recommendations for the prevention and treatment of overweight and obesity in adults
(FESNAD-SEEDO consensus document). The role of diet in obesity treatment (III/III). Nutr Hosp 2012; 27(3): 833-864.

7. DeLorenzo A, Fidanza F. La dieta di Nicotera nel 1960. Dieta Mediterranea Italiana di Riferimento. EMSI Editore 2006; 1-75.

8. Keys A, Menotti A, Arvanis C, et al. The Seven Countries Study: 2.289 death in 15 years. Prev Med 1984; 13: 141-154.

9. DelVillar G M, Chillerón S C, Diaz-Gutierrez J, et al. Healthy Lifestyle and Incidence of Metabolic Syndrome in the SUN Cohort. Nutrients 2018; 11(1).

10. 10. Danesh J. EPIC-Heart: The cardiovascular component of a prospective study of nutritional, lifestyle and biological factors in 520,000 middleaged participants from 10 European countries. European Journal of Epidemiology 2007; 22(2): 129-141.

11. Kris-Etherton $P$, Eckel $R H$, Howard $B ~ V$, et al. AHA Science Advisory: Lyon Diet Heart Study. Benefits of a Mediterranean-style, National Cholesterol Education Program/American Heart Association Step I Dietary Pattern on Cardiovascular Disease. Circulation 2001; 103(13): 1823-5.

12. Marchioli R, Barzi F, Bomba E, et al. Early Protection Against Sudden Death by n-3 Polyunsaturated Fatty Acids After Myocardial Infarction Time-Course Analysis of the Results of the Gruppo Italiano per lo Studio della Sopravvivenza nell'Infarto Miocardico (GISSI)-Prevenzione. Circulation 2002; 105(16): 1897-1903.

13. Catapano A L, Graham I, De Backer G, et al. 2016 ESC/EAS Guidelines for the Management of Dyslipidaemias. Eur Heart J. 2016 Oct 14; 37(39): 2999-3058.

14. Zambon A, Marchiori M, Manzato E. Obesità viscerale e dislipidemia: meccanismi fisiopatologici, implicazioni cliniche e terapia. Giornale Italiano di Cardiologia 2008; 9: 1-4.

15. Schaefer E J. Lipoproteins, nutrition, and heart disease. The American journal of clinical nutrition 2002; 75(2): 191-212.

16. Corsetti J P, Bakker SJL, Gansevoort R T, et al. Compositional Features of HDL Particles Interact with Albuminuria to Modulate Cardiovascular Disease Risk. Int J Mol Sci 2019; 20(4).

17. 17. Krauss RM, Siri PW. Metabolic abnormalities: triglyceride and low-density lipoprotein. Endocrinology and Metabolism Clinics of North America 2004; 33(2): 405-415.

18. Packard C, Caslake M, Shepherd J. The role of small, dense low density lipoprotein (LDL): a new look. International Journal of Cardiology 2004; 74 : S17-S22.

19. Fuentes F, López-Miranda J, Sánchez E, et al. Mediterranean and low-fat diets improve endothelial 
function in hypercholesterolemic men. Annals of Internal Medicine 2001; 134(12): 1115-1119.

20. Asgari S, Abdi H, Hezaveh A M, Moghisi A, et al. The Burden of Statin Therapy based on ACC/AHA and NCEP ATP-III Guidelines: An Iranian Survey of Non-Communicable Diseases Risk Factors. Sci Rep. 2018; 8(1): 4928.

21. 21. Simopoulos, A P. The importance of the ratio of omega-6/omega-3 essential fatty acids. Biomedicine e Pharmacotherapy 2002; 56(8), 365-379.

22. Gammone M A, Riccioni G, D'Orazio N. Carotenoids: Potential allies of cardiovascular health? Food Nutr. Res. 2015; 59: 26762.

23. Riccioni G, Gammone M A, Tettamanti G, et al. Resveratrol and anti-atherogenic effects. Int. J. Food Sci. Nutr. 2015; 66: 603-610.

24. Gammone M A, Efthymakis K, Pluchinotta F R, et al. Impact of chocolate on the cardiovascular health. Front. Biosci. (Landmark Ed.) 2018; 23: 852-864.

25. Gammone M A, Tettamanti G, Bergante S, et al. Prevention of cardiovascular diseases with carotenoids. Front. Biosci. (Schol. Ed.) 2017; 9: 165-171.

26. Gammone M A. Carotenoids, ROS, and cardiovascular health (Book Chapter). Reactive Oxygen Species in Biology and Human Health 2017; 1: 325-331.

27. Visioli F, Bernardini E. Extra Virgin Olive Oils Polyphenols: Biological Activities. Current pharmaceutical design 2011; 17(8): 786-804.

28. Gammone M A, Riccioni G, Parrinello G, et al. Omega-3 polyunsaturated fatty acids: Benefits and endpoints in sport. Nutrients 2019; 11(1): 46.

29. Gammone M A, Gemello E, Riccioni, G, et al. Marine bioactives and potential application in sports. Mar. Drugs 2014; 12: 2357-2382.

30. D'Orazio N, Gammone M A, Gemello E, et al. Marine bioactives: Pharmacological properties and potential applications against inflammatory diseases. Mar. Drugs 2012; 10: 812-833.

31. Gammone M A, D'Orazio N. Anti-obesity activity of the marine carotenoid fucoxanthin. Mar. Drugs 2015; 13: 2196-2214.

32. Gammone M A, Riccioni G, Galvano F, et al. Novel Therapeutic Strategies Against Cancer: Marinederived Drugs May Be the Answer? Anticancer Agents Med. Chem. 2016; 16: 1549-1557.

33. Gammone M A, Riccioni G, D'Orazio, N. Marine carotenoids against oxidative stress: Effects on human health. Mar. Drugs 2015; 13: 6226-6246.

34. D'Orazio N, Gemello E, Gammone M A, et al. Fucoxantin: A treasure from the sea. Marine Drugs 2012; 10(3): 604-616.

35. National Cholesterol Education Program (NCEP) Expert Panel on Detection, Evaluation, and Treatment of High Blood Cholesterol in Adults (Adult Treatment Panel III). Third Report of the NCEP Expert Panel on Detection, Evaluation, and
Treatment of High Blood Cholesterol in Adults (Adult Treatment Panel III) final report. Circulation. 2002; 106(25): 3143-421.

36. Kurisu S, Ikenaga $\mathrm{H}$, Watanabe $\mathrm{N}$, et al. Implications of World Health Organization classification for body mass index on the correlations between common electrocardiographic indexes for left ventricular hypertrophy and left ventricular mass. Clin Exp Hypertens. 2016; 38(8): 715-720.

37. Martone D, Roccaldo R, Censi L, et al. Food consumption and nutrient intake in Italian school children: results of the ZOOM8 study. Int J Food Sci Nutr. 2013; 64(6): 700-5.

38. Hsu P H, Lee C H, Kuo L K, et al. Determination of the energy requirements in mechanically ventilated critically ill elderly patients in different BMI groups using the Harris-Benedict equation. J Formos Med Assoc. 2018; 117(4): 301-307.

39. Tsujimoto $T$, Kajio $H$. Abdominal Obesity Is Associated With an Increased Risk of All-Cause Mortality in Patients with HFpEF. J Am Coll Cardiol. 2017; 70(22): 2739-2749.

40. Sapunar J, Aguilar-Farías N, Navarro J, et al. High prevalence of dyslipidemia and high atherogenic index of plasma in children and adolescents. Rev Med Chil. 2018; 146(10): 1112-1122.

41. Martínez-González M A, Gea A, Ruiz-Canela M. The Mediterranean Diet and Cardiovascular Health. Circ Res. 2019; 124(5): 779-798.

42. Hennein R, Liu C, McKeown N M, et al. Increased Diet Quality is Associated with Long-Term Reduction of Abdominal and Pericardial Fat. Obesity (Silver Spring) 2019; 1.

43. Riccioni G, Gammone M A, Currenti W, et al. Effectiveness and safety of dietetic supplementation of a new nutraceutical on lipid profile and serum inflammation biomarkers in hypercholesterolemic patients. Molecules 2018; 23: 1168.

44. Gammone MA, Vicentini A, Riccioni G, et al. FoodRelated Atrial Fibrillation? The Potential Role of Biogenic Amines in "Nutri-Arrhythmias" Genesis. Reports 2019, 2(1), 1. 